\title{
Novel Temperature Characteristics of Gain Behaviors in Quantum-Dot Lasers
}

\author{
Yi Ho, Tzeng Wei-Chieh and Ching-Fuh Lin* \\ Graduate institute of Electro-Optical Engineering, National Taiwan University, \\ No.1, Sec. 4, Roosevelt Road, Taipei, 106, Taiwan, R.O.C \\ *also with Graduate institute of Electronics Engineering and Dept. of Electrical Engineering \\ cflin@cc.ee.ntu.edu.tw
}

\begin{abstract}
In quantum dots, the increment of temperature results in red shift of gain spectrum. Thermal state-filling and electron-phonon scattering lead to extremely large and even negative $T_{0}$. Theoretical prediction is confirmed experimentally.
\end{abstract}

\section{INTRODUCTION}

Quantum dot is noted for the low threshold current, high differential gain and high characteristics temperature which are good for applications. [1]

\section{DEPENDENCE OF GAIN SPECTRUM ON}

\section{TEMPERATURE}

There are three main causes of dependence of peak wavelength on quantum dot (QD) laser diodes: thermal expansion, thermal state-filling, and non-equilibrium scattering. First, thermal expansion results in decrease of bandgap energy and red shift. The general expression for bandgap dependence around room temperature with small deviation is given by:

$$
E_{g}(T)=E_{g 0}-\frac{\alpha T^{2}}{T+\beta} \ldots . .(1)
$$

where the numerical values for InAs are given by [2].

Second, thermal state-filling causes blue shift . More thermal energy provides higher probability for electrons reside in higher energy levels. The last, non-equilibrium scattering results in blue shift of the spectrum of lower quantized states. In quantum dots, transition of strongly-localized electrons involved with phonon can let electrons relax to lower quantized states. With higher temperature, such electron-phonon scattering can increase the carrier density of lower quantized states and cause blue shifts of them, in the meanwhile, decrease the carrier density of higher quantized states and cause red shifts of them.

\section{SCATTERING DEPENDENT ON TEMPERATURE}

The capture and relaxation time are determined by electron-phonon scattering with rates as follows:

$$
\frac{1}{\tau}=\frac{2 \pi}{\hbar} \int\left|M_{q}^{i f}\right|^{2}\left(N_{q}+1\right) \delta\left(E_{i f}-\hbar \omega_{q}\right) d^{3} q
$$

However, the dependence of scattering rates can be expressed in power series in positive powers. Consider operation around room temperature with slight variance of temperature, we can approximate the scattering ratesare inversely proportional to $\mathrm{T}$. $[4]$

\section{NON-EQUILIBRIUM FERMI-DIRAC DISTRIBUTION}

When the carrier capture and relaxation is limited in QD, the distribution of electrons which doesn't follow Fermi-Dirac distribution is given by: [3]

$$
\begin{gathered}
f\left(E_{i}\right)=\frac{1}{1+R^{*} \exp \left(\frac{E_{i}-E_{f}}{k_{B} T}\right)} \\
\text { where } R=\tau_{c, r}\left(\frac{1}{\tau_{e}}+\frac{1}{\tau_{s}}\right)<1
\end{gathered}
$$

where $\tau_{c, r}$ is overall capture/relaxation lifetime, into/in QD, $\tau_{e}$ is stimulated emision lifetime out of $\mathrm{QD}$, and $\tau_{s}$ is carrier recombination lifetime. The factor $\mathrm{R}<1$ shows that the phonon bottleneck can lift the Fermi level and increase the probability for electrons in higher energy levels. The gain. is given by :

$$
g(\hbar \omega)=\frac{2 N_{D}}{W} \int \sigma_{g}\left(\hbar \omega, E_{i}\right) g_{\text {inh }}\left(E_{i}\right)\left[2 f\left(E_{i}\right)-1\right] d E_{i} \cdots
$$

With small deviation in temperature, the dependence of $\tau_{e}$ and $\tau_{c}$ can be approximated as linear with $\mathrm{T}$ and $\tau_{s}$ as a constant. So the shift of quasi-Fermi level is given by

$$
\Delta E_{f}(\Delta T)=\Delta E_{g}-k_{B} T \ln (1-c \Delta T)
$$

On the right hand of the equation, with $\Delta T>0$ the first term is negative and the second is positive. At certain temperature, we can find either blue or red shift will happen at certain photon wavelength.

In Fig 1, theoretical calculations are performed by assuming a QD carrier density of $5^{*} 10^{12} \mathrm{~cm}^{-2}$ in injector-well structure layer. We can find the peak gain and peak photon energy decrease with increment of temperature. The thermal expansion of the lattice dominates the thermal band-filling effect and the scattering of phonons and electrons from higher energy levels. We find red shift finally. The peak gain decreases with increment of temperature because of lattice expansion. 


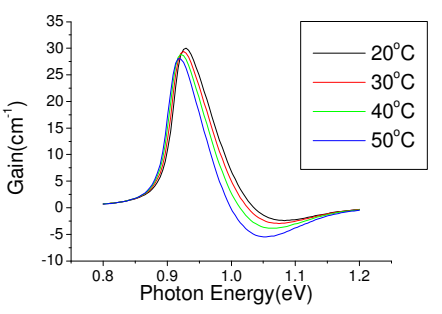

Fig 1. Theoretical Calculation of

Gain for $1^{\text {st }}$ Quantized State Only

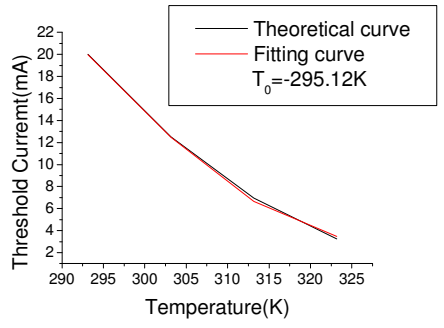

Fig 2. Theoretical Calculation of

Threshold Current

In Fig 2., we can find $\mathrm{T}_{0}<0$ for photon energy smaller than the peak photon energy. For ground state $T_{0}$ around the peak photon energy approximate infinitely large and even negative such as $-295.12 \mathrm{~K}$ at photon energy $0.9 \mathrm{eV}$.

\section{EXPERIMENTAL RESULTS}

In Fig 3, red shift with increasing temperature is observed as predicted by the simulation. The $1^{\text {st }}$ and $2^{\text {nd }}$ quantized states lase at photon energy $0.98 \mathrm{eV}$ and $1.1 \mathrm{eV}$ respectively.

In Fig 4., we can find the threshold current decrease with increment of temperature at the photon energy $0.973 \mathrm{eV}$. Negative temperature effect is observed with different positive and even negative $\mathrm{T}_{0}$ values at different temperatures.

\section{CONCLUSION}

In quantum dot, the increment of temperature results in red shift by thermal expansion which dominates the blue shift by thermal band-filling and electron-phonon scattering. The latter two causes bring about improved temperature characteristics with extremely large positive $\mathrm{T}_{0}$ and even negative $T_{0}$ around the peak photon energy theoretically and experimentally.

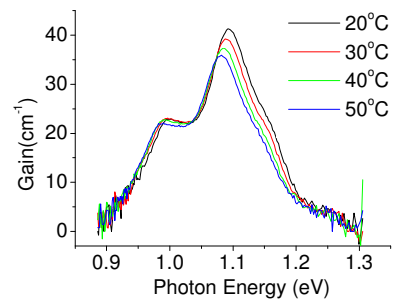

Fig 3. Experimental Gain curves

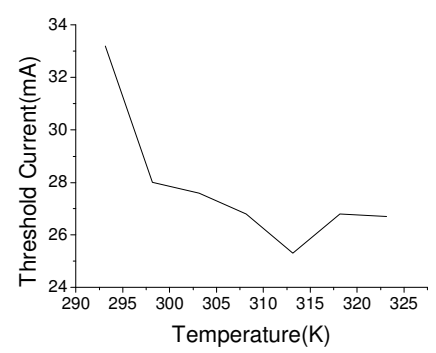

Fig 4. Experimental Threshold Current

ACKNOWLEDGEMENT

This research is supported by NSC93-2120-M-002-011 .

\section{REFERENCES}

[1] "Carrier Dynamics and High-Speed Modulation Properties of Tunnel Injection InGaAs-GaAs Quantum-Dot Lasers", Pallab Bhattacharya Siddhartha Ghosh, Sameer Pradhan, Jasprit Singh, Zong-Kwei Wu, J. Urayama, Kyoungsik Kim, and Theodore B. Norris, IEEE Journal of Quantum Electronics, Vol. 39, No. 8, AUG 2003

[2] "Temperature dependence of the photoluminescence emission from InAs quantum dots in a strained Ga0.85In0.15As quantum well", Dan P Popescu, Peter G Eliseev, Andreas Stintz and Kevin J Malloy, Semicond. Sci. Technol. 19 (2004) 33-38 PII: S0268-1242(04)63380-3

[3]"Nonequilibrium distribution in quantum dots lasers and influence on laser spectral output”H. Jiang and J. Singh, J. OF App. Phys. Vol 85, Num. 10, 1999

[4] "Evalution of Hole drift mobility in glassy As2S3 in the temperature range 77 - 330 K, A. M. Andriesh, I. P. Culeac, P. J. S. Ewen, A. E. Owen , Journal of Optoelectronics and Advanced Materials Vol. 3, No. 1, March 2001, p. 27-31 